\title{
CLASS B1555+375: A NEW FOUR-IMAGE GRAVITATIONAL LENS SYSTEM ${ }^{1}$
}

\author{
D. R. Marlow, S. T. Myers, AND D. Rusin \\ Department of Physics and Astronomy, University of Pennsylvania, 209 South 33d Street, Philadelphia, PA 19104 \\ N. Jackson, I. W. A. Browne, P. N. Wilkinson, and T. Muxlow \\ Nuffield Radio Astronomy Laboratories, Jodrell Bank, University of Manchester, Macclesfield, Cheshire SK11 9DL, UK \\ C. D. FASSNACHT \\ National Radio Astronomy Observatory, P.O. Box O, Socorro, NM 87801 \\ L. Lubin, T. Kundić, R. D. Blandford, T. J. Pearson, and A. C. S. Readhead \\ Palomar Observatory, California Institute of Technology, Mail Stop 105-24, Pasadena, CA 91125 \\ L. KOOPMANS \\ Kapteyn Astronomical Institute, Postbus 800, NL-9700 AV Groningen, Netherlands \\ AND \\ A. G. DE BRUYN ${ }^{2}$ \\ Netherlands Foundation for Research in Astronomy, Postbus 2, NL-7990 AA Dwingeloo, Netherlands \\ Received 1999 April 7; accepted 1999 April 29
}

\begin{abstract}
We have discovered a new gravitational lens in the Cosmic Lens All-Sky Survey. The lens B1555+375 is a four-image system with a maximum separation of 0"42. VLA and MERLIN radio observations show these images in a characteristic quadruple-lens configuration. Optical imaging with the Keck II Telescope at $R$ band shows a faint extended object. We estimate the combined emission from the lens and background source to be $R=25$ mag. Observations at $H$ band with the William Herschel Telescope also detected this extended object. The combined lens and background source magnitude was measured to be $H=19$ mag. Presently, redshifts for both the lensing galaxy and background source are undetermined. We conclude that one or more of the following are true: the lens galaxy is sub- $L^{*}$, it has $z>0.5$, or it is highly reddened. The observed MERLIN component positions and flux densities can be well described by a lens model based on a singular isothermal ellipsoid mass distribution. Our best-fit lens model has a reduced $\chi^{2}$ of 2.6. High-resolution VLA radio observations should help to constrain the lens model further.

Key words: gravitational lensing
\end{abstract}

\section{INTRODUCTION}

The Cosmic Lens All-Sky Survey (CLASS) aims to find new cases of gravitational lensing among flat-spectrum radio sources (Myers et al. 1995, 1999b). CLASS builds upon the success of the earlier Jodrell Bank-VLA ${ }^{3}$ Astrometric Survey (JVAS; Patnaik et al. 1992; Browne et al. 1998; Wilkinson et al. 1998) and extends the search to weaker flux densities. The primary goals of the survey are to find lens systems suitable for determination of the Hubble constant, $H_{0}$ (Refsdal 1964; Blandford \& Narayan 1992) and to study the frequency of lensing in order to place constraints on the

\footnotetext{
${ }^{1}$ Based on observations made with the National Radio Astronomy Observatory (NRAO), which is a facility of the National Science Foundation operated under cooperative agreement by Associated Universities, Inc.; with the Multi-Element Radio-linked Interferometer Network (MERLIN), which is a national facility operated by the Nuffield Radio Astronomy Laboratories, Jodrell Bank, University of Manchester, on behalf of the UK Particle Physics and Astronomy Research Council; with the William Herschel Telescope (WHT), which is operated on the island of La Palma by the Isaac Newton Group in the Spanish Observatorio del Roque de los Muchachos of the Instituto de Astrofísica de Canarias; and with the W. M. Keck Observatory, which is operated as a scientific partnership among the California Institute of Technology, the University of California, and the National Aeronautics and Space Administration. The Keck Observatory was made possible by the generous financial support of the W. M. Keck Foundation.

${ }^{2}$ Also Kapteyn Astronomical Institute, Postbus 800, NL-9700 AV Groningen, Netherlands.

${ }^{3}$ Very Large Array is operated by NRAO.
}

value of the cosmological constant $\Lambda$ (Quast \& Helbig 1999; Helbig et al. 1999; Falco, Kochanek, \& Muñoz 1998).

To date, CLASS has observed over $\sim 12,000$ sources with the VLA in the A configuration at $8.4 \mathrm{GHz}$. The observations have been performed over three phases (CLASS 1-3) from spring 1994 to summer 1998. Sources in the first two phases of CLASS were selected from the 87GB survey at $5 \mathrm{GHz}$ (Gregory \& Condon 1991) with $S_{5} \geq 25 \mathrm{mJy}$ and with spectral index $\alpha \geq-0.5$ (where $S_{v} \propto v^{\alpha}$ ) between 5 $\mathrm{GHz}$ and the $325 \mathrm{MHz}$ Westerbork Northern Sky Survey (Rengelink et al. 1997) or the $365 \mathrm{MHz}$ Texas Survey (Douglas et al. 1996). Recently, a reselection of the sources in CLASS was made using the GB6 survey (Gregory et al. 1996) and the NRAO VLA Sky Survey (Condon et al. 1998).

The VLA survey data were calibrated within AIPS using the usual procedure. The sources were mapped using an automatic script within the imaging package DIFMAP (Shepherd 1997). The data were then modeled with Gaussian components, providing a quantitative way of selecting lensed compact components. The aim of CLASS is to find all cases of lensing of compact radio cores with component separations $0 " 3 \leq \Delta \theta \leq 6{ }^{\prime \prime} .0$ and flux density ratios $\leq 10: 1$. Flat-spectrum radio sources tend to be dominated by emission from a single compact core; those sources within CLASS having multiple compact components are selected as lens candidates. These candidates are followed up with high-resolution radio observations using MERLIN and then the VLBA for the few surviving sources. The vast 
majority of the lens candidates are rejected on surface brightness and morphological grounds. Those candidates surviving the radio filter are followed up further with optical and/or near-infrared imaging and spectroscopy.

The first phase of CLASS observations (CLASS 1) has yielded four new lens systems: B0712 +472 (Jackson et al. 1998b), B1600 + 434 (Jackson et al. 1995), B1608 +656 (Myers et al. 1995; Fassnacht et al. 1996), and B1933 +503 (Sykes et al. 1998). The second series of observations (CLASS 2) has until now produced two new lens systems: B2045+265 (Fassnacht et al. 1999) and B1127+384 (Koopmans et al. 1999). Here we report the discovery of another CLASS 2 lens, B1555+375, found during MERLIN follow-up observations of lens candidates. Two additional lenses have also been discovered recently during the third phase of CLASS observations (CLASS 3): B1152 + 199 and B1359+ 154 (Myers et al. 1999a).

\section{RADIO OBSERVATIONS}

The CLASS observation of B1555 + 375 was made on 1995 August 14 as part of CLASS 2. The system was observed at $8.4 \mathrm{GHz}$ with the VLA in the A configuration, giving a resolution of $\sim 0$ ".25. The observation and data reduction techniques for the survey are discussed in Myers et al. (1999b). The final map (Fig. 1) shows an extended object consisting of two brightness peaks and a faint $(3 \sigma)$ component to the south. The extended object was modeled by two compact Gaussian components with a total flux density of $45.7 \mathrm{mJy}$ and a flux density ratio of $3.7: 1$. The relative positional uncertainties for the different components are estimated as the beam size at each frequency divided by the signal-to-noise ratio of the component flux densities in the map (see Table 1).

The system was observed with MERLIN $5 \mathrm{GHz}$ snapshot observations on 1996 December 26. The total integration time was $1 \mathrm{hr}$. The final map (not shown) has an rms

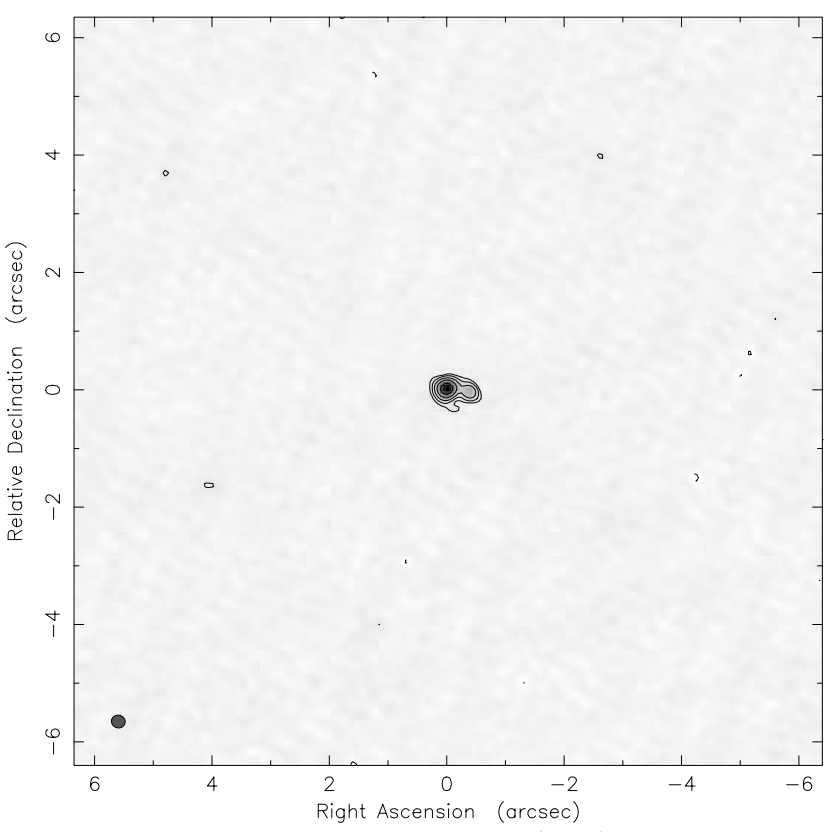

FIG. 1.-VLA $8.4 \mathrm{GHz}$ observation of $\mathrm{B} 1555+375$ taken 1995 August 14. Contours are plotted at $(-3,3,6,12,24,48) \times 0.417$ mJy beam $^{-1}$ with a map peak flux density of $31.9 \mathrm{mJy}^{\text {beam }}{ }^{-1}$. The beam is $0^{\prime \prime} .234 \times 0^{\prime \prime} .217$ at 73.1. The map is centered on (J2000.0) R.A. $15^{\mathrm{h}} 57^{\mathrm{m}} 11^{\mathrm{s}} .94$ decl. $+37^{\circ} 21^{\prime} 35^{\prime \prime} .95$.
TABLE 1

VLA $8.4 \mathrm{GHz}$ Model-Fit Component Positions AND Flux DENSITIES

\begin{tabular}{lcccr}
\hline \hline & \multicolumn{2}{c}{ OfFSET $(\operatorname{arcsec})$} & & \\
\cline { 2 - 3 } COMPONENT & East & North & $\begin{array}{c}\sigma_{x} \\
(\operatorname{arcsec})\end{array}$ & $\begin{array}{c}S_{8.4} \\
(\mathrm{mJy})\end{array}$ \\
\hline $\mathrm{A} \ldots \ldots \ldots \ldots$ & 0 & 0 & $\ldots$ & 36.1 \\
$\mathrm{~B} \ldots \ldots \ldots \ldots$ & -0.3863 & -0.0510 & \pm 0.010 & 9.6 \\
\hline
\end{tabular}

NotE.-Positions are offset from (J2000.0) R.A. $15^{\mathrm{h}} 57^{\mathrm{m}} 11^{\mathrm{s}} \mathrm{s} .9399$ decl. $+37^{\circ} 21^{\prime} 35^{\prime \prime} .965$. The rms noise level in the map is $140 \mu \mathrm{Jy}_{\text {beam }^{-1}}$.

noise level of $180 \mu \mathrm{Jy}$ beam ${ }^{-1}$ and an angular resolution of 50 mas. The data were modeled by three point sources with a total flux density of $34.9 \mathrm{mJy}$ (note that B1555 + 375 was selected from the $87 \mathrm{~GB}$ survey with a $5 \mathrm{GHz}$ flux density of $30 \mathrm{mJy}$ ). Indications of a possible fourth component at the 3 $\sigma$ level were detected in the map. The relative positions of the image components were consistent with that expected for a four-image lens system. In order to obtain a map with improved sensitivity and to investigate the possibility of a fourth component, the system was reobserved with MERLIN at $5 \mathrm{GHz}$ on 1997 December 1 with a total integration time of $16 \mathrm{hr}$ on source. The final map (Fig. 2) has an rms noise level of $60 \mu \mathrm{Jy}$ beam $^{-1}$ and an angular resolution of 50 mas. The data were modeled by four Gaussian components (named clockwise A-D) with a total flux density of $36.3 \mathrm{mJy}$ (Table 2). These components were arranged in a characteristic "quad lens" configuration (see the CLASS lens B1608 + 656 for a good example; Myers et al. 1995).

A follow-up VLA $15 \mathrm{GHz}$ A-configuration snapshot was made on 1998 May 15 with a total integration time of 5 minutes. The rms noise level was $270 \mu \mathrm{Jy}$ beam $^{-1}$, and the

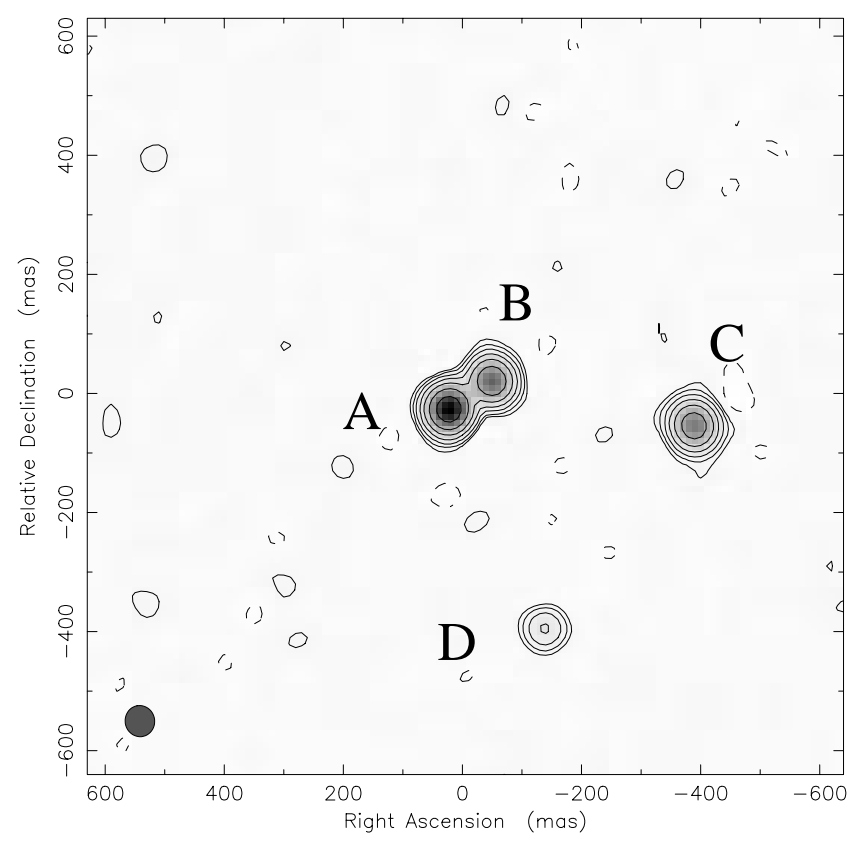

FIG. 2.-MERLIN $5 \mathrm{GHz}$ observation of B1555+375 taken 1997 December 1 . The individual lensed images are labeled clockwise A-D. Contours are plotted at $-1 \%, 1 \%, 2 \%, 4 \%, 8 \%, 16 \%, 32 \%$, and $64 \%$ of the map peak flux density of $16.3 \mathrm{mJy}^{\text {beam }}{ }^{-1}$. The beam is 52.5 mas $\times$ 49.5 mas at $10^{\circ} .7$. The map is centered on (B1950.0) R.A. $15^{\mathrm{h}} 55^{\mathrm{m}} 22^{\mathrm{s}} .030$ decl. $+37^{\circ} 30^{\prime} 10^{\prime \prime} 942$ 
TABLE 2

MERLIN $5 \mathrm{GHz}$ Model-Fit CoMponent Positions AND Flux Densities

\begin{tabular}{lcccc}
\hline \hline & \multicolumn{2}{c}{ OfFSET (arcsec) } & & \\
\cline { 2 - 3 } CoMPONENT & East & North & $\begin{array}{c}\sigma_{x} \\
(\operatorname{arcsec})\end{array}$ & $\begin{array}{c}S_{5} \\
(\mathrm{mJy})\end{array}$ \\
\hline A $\ldots \ldots \ldots \ldots$ & 0 & 0 & $\ldots$ & 17.0 \\
B $\ldots \ldots \ldots \ldots$. & -0.0726 & +0.0480 & \pm 0.001 & 9.7 \\
C $\ldots \ldots \ldots \ldots$ & -0.4117 & -0.0280 & \pm 0.001 & 8.3 \\
D $\ldots \ldots \ldots \ldots$ & -0.1619 & -0.3680 & \pm 0.006 & 1.3 \\
\hline
\end{tabular}

Note.-Data for the 1997 December 1 observation. Positions are offset from (B1950.0) R.A. $15^{\mathrm{h}} 55^{\mathrm{m}} 22^{\mathrm{s}} .02982$ decl. $+37^{\circ} 30^{\prime} 10^{\prime \prime} 94237$. The rms noise level in the map is $60 \mu \mathrm{Jy}$ beam $^{-1}$.

angular resolution was 150 mas. The peak flux density in the map was $34 \mathrm{mJy}^{\text {beam }}{ }^{-1}$. The data were modeled by four Gaussian components with a total flux density of 55.7 mJy (Table 3). Components A and B were not fully resolved in the VLA $15 \mathrm{GHz}$ map (Fig. 3); in other words, com-

TABLE 3

VLA $15 \mathrm{GHz}$ Model-Fit Component Positions AND Flux DENSITIES

\begin{tabular}{lcccc}
\hline \hline & \multicolumn{2}{c}{ OfFSET $(\operatorname{arcsec})$} & & \\
\cline { 2 - 3 } CoMPONENT & East & North & $\begin{array}{c}\sigma_{x} \\
(\operatorname{arcsec})\end{array}$ & $\begin{array}{c}S_{15} \\
(\mathrm{mJy})\end{array}$ \\
\hline $\mathrm{A} \ldots \ldots \ldots \ldots$ & 0 & 0 & $\ldots$ & 27.0 \\
$\mathrm{~B} \ldots \ldots \ldots \ldots$ & -0.0727 & +0.0490 & \pm 0.010 & 15.2 \\
$\mathrm{C} \ldots \ldots \ldots \ldots$ & -0.4137 & -0.0280 & \pm 0.012 & 11.4 \\
$\mathrm{D} \ldots \ldots \ldots \ldots$ & -0.1586 & -0.3480 & \pm 0.055 & 2.1 \\
\hline
\end{tabular}

Note.-Positions are offset from (J2000.0) R.A. $15^{\mathrm{h}} 57^{\mathrm{m}} 11^{\mathrm{s}} \mathrm{s} 9450 \mathrm{decl}$. $+37^{\circ} 21^{\prime} 35^{\prime \prime} .934$. The rms noise level in the map is $270 \mu \mathrm{Jy}_{\text {beam }}^{-1}$.

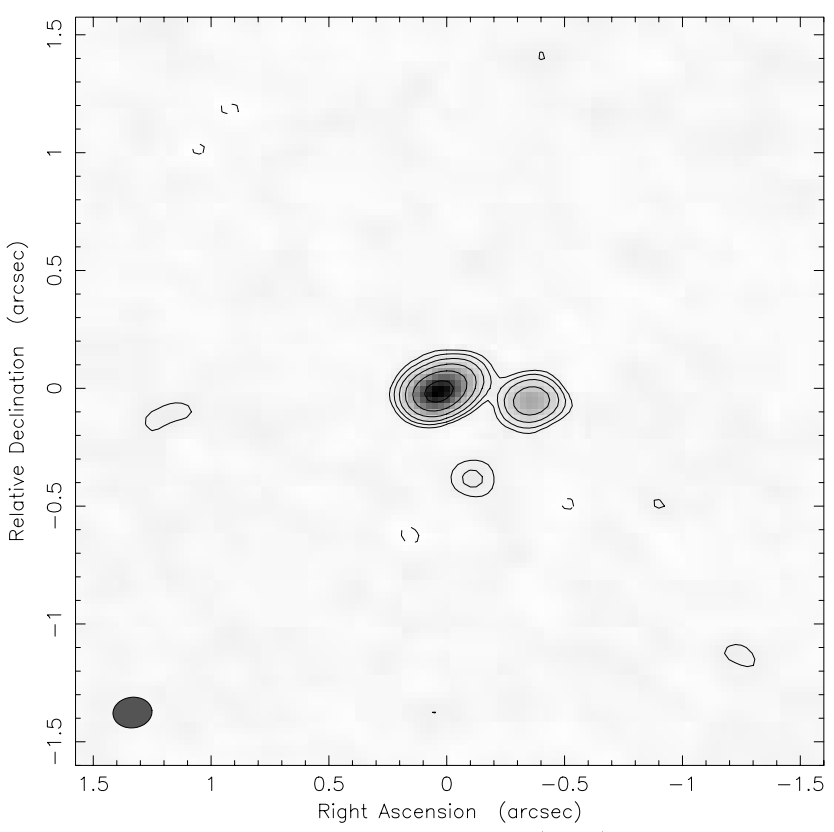

FIG. 3.-VLA $15 \mathrm{GHz}$ observation of B1555+375 taken 1998 May 15. Contours are plotted at $-2.4 \%, 2.4 \%, 4.8 \%, 9.6 \%, 19.2 \%, 38.4 \%$, and $76.8 \%$ of the map peak flux density of $34.0 \mathrm{mJy}^{\text {beam }}{ }^{-1}$. The beam is $0.166 \times 0^{\prime \prime} .13$ at $-83^{\circ} .4$. The map is centered on (J2000.0) R.A. $15^{\mathrm{h}} 57^{\mathrm{m}} 11^{\mathrm{s}} .940$ decl. $+37^{\circ} 21^{\prime} 35^{\prime \prime} .965$. ponent spectra cannot be reliably formed. However, from total flux measurements, the lensed source appears to have an inverted spectrum $\left(\alpha_{5}^{15} \sim+0.39\right)$. Further multifrequency, high-resolution radio observations are required to determine the component spectra and to search for signs of variability.

\section{OPTICAL AND NEAR-INFRARED IMAGING}

B1555+ 375 was observed on 1997 August 24 with the William Herschel Telescope (WHT) using the WHT Infrared Camera. Five exposures were obtained, totaling 20 minutes at $H$ band. The images (Fig. 4) were reduced using standard analysis tools in IRAF. ${ }^{4}$ Faint extended emission coincident with the radio position was detected within the astrometric errors. The integrated emission from both the lensed images and the lensing galaxy gave a magnitude of $H \sim 19 \mathrm{mag}$. There is a bright region to the north of the $H$-band object that may be associated with the two close lensed images seen in the radio observations. No further sign of emission was detected within $10^{\prime \prime}$ of the target.

Subsequently, B1555 + 375 was imaged on 1998 August 2 using the Low Resolution Imaging Spectrograph (LRIS; Oke et al. 1995) on the Keck II Telescope. The observing conditions were not ideal; the observations were obtained through cirrus and were contaminated by moonlight. Two 5 minute exposures were obtained through the $R$-band filter. These images were reduced using standard tasks in IRAF, and then they were co-added. A faint extended object was detected within the astrometric errors at the position of the

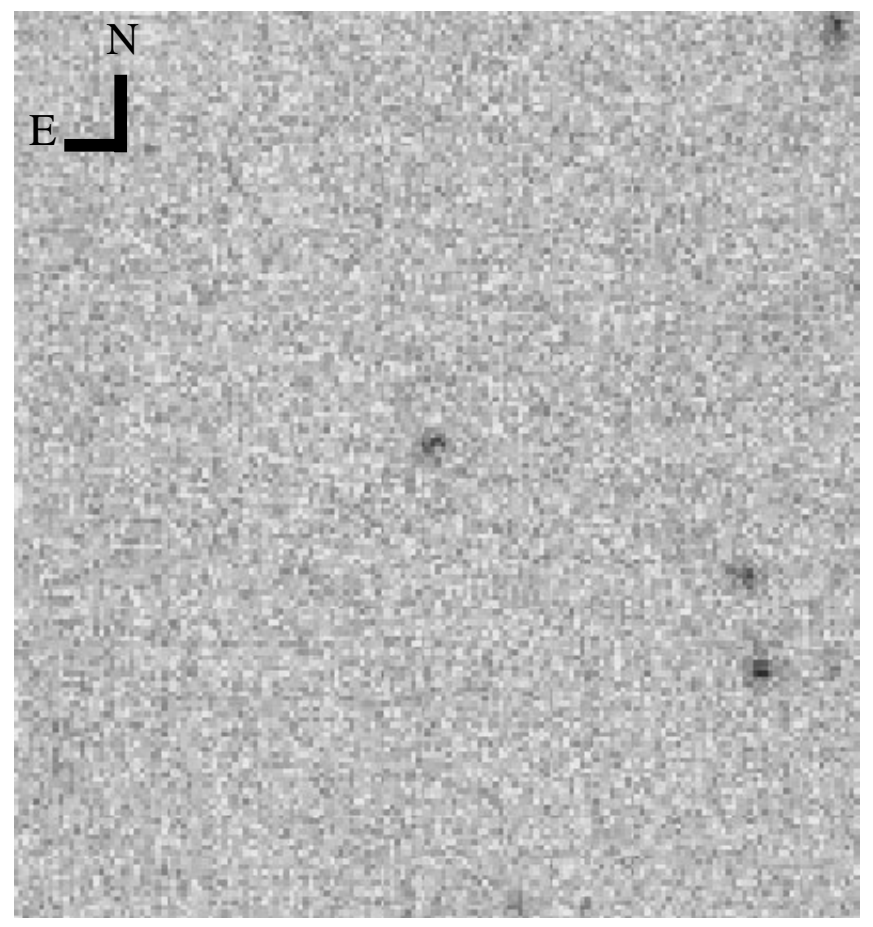

FIG. 4.-WHT $H$-band image of B1555 +375 . A faint extended object is located at the center of the image, and we identify this with the B1555+375 system. We estimate a combined (lens and background source) magnitude of $H=19 \mathrm{mag}$. The image size is approximately $50^{\prime \prime} \times 50^{\prime \prime}$ with a $0^{\prime \prime} .24$ pixel size.

${ }^{4}$ IRAF is distributed by the National Optical Astronomy Observatories, which are operated by the Association of Universities for Research in Astronomy, Inc., under cooperative agreement with NSF. 


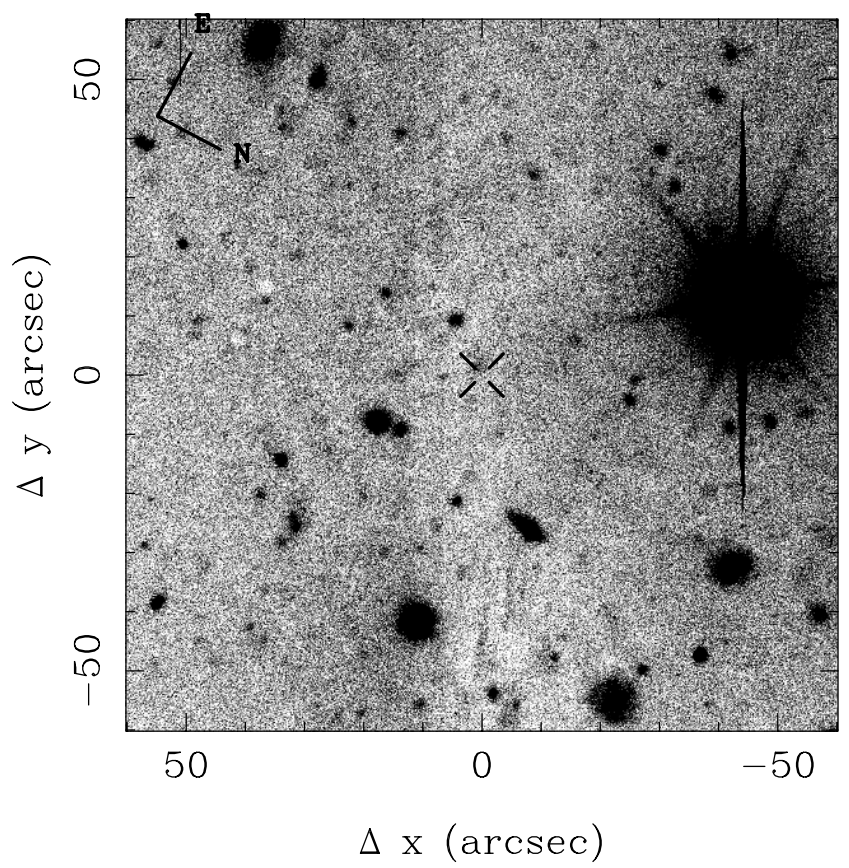

FIG. 5.-LRIS $R$-band image of $\mathrm{B} 1555+375$. A faint extended object is located above the crosshair, and we identify this with the B1555+375 system. We estimate a combined (lens and background source) magnitude of $R=25$ mag within a 0.86 aperture.

B1555 + 375 radio emission (Fig. 5). We estimate that the combined emission from the deflector and background source produces a magnitude of $R=25$ mag within a 0 ".86 diameter aperture.

If the lens has the luminosity of an elliptical $L^{*}$ galaxy, we would expect it to have $R=20.3 \mathrm{mag}$ for $z_{l}=0.5$ (including a $K$-correction but no evolutionary correction; Poggianti 1997). ${ }^{5}$ At $H$ band we would expect $H=17.5$ mag. Since we measure the combined light from the lens and lensed images to be $R=25 \mathrm{mag}$ and $H=19 \mathrm{mag}$, we must assume one or

TABLE 4

Model Lens AND Source Parameters for B1555 + 375

\begin{tabular}{lccccc}
\hline \hline & \multicolumn{2}{c}{ OffSET $(\operatorname{arcsec})$} & & & \\
\cline { 2 - 3 } CoMPONENT & East & North & $\begin{array}{c}\sigma \\
\left(\mathrm{km} \mathrm{s}^{-1}\right)\end{array}$ & $\begin{array}{c}\text { P.A. } \\
(\mathrm{deg})\end{array}$ & $b / a$ \\
\hline Lens ........ & -0.1651 & -0.2403 & 133.394 & 1.9689 & 0.4543 \\
Source...... & -0.1801 & -0.1532 & $\ldots$ & $\ldots$ & $\ldots$ \\
\hline
\end{tabular}

TABLE 5

Model-Predicted Image Positions, Magnifications, AND Time DELAYS FOR B1555+375

\begin{tabular}{lcccc}
\hline \hline & \multicolumn{2}{c}{ OffSET $(\operatorname{arcsec})$} & & \\
\cline { 2 - 3 } CoMPONENT & East & North & $\mu$ & $\begin{array}{c}\Delta t \\
\left(h^{-1} \text { days }\right)\end{array}$ \\
\hline A $\ldots \ldots \ldots \ldots$ & +0.0039 & +0.0056 & +8.20 & +0.48 \\
B $\ldots \ldots \ldots \ldots$. & -0.0753 & +0.0436 & -7.64 & +0.49 \\
C $\ldots \ldots \ldots \ldots$ & -0.4127 & -0.0322 & +2.78 & +0.00 \\
D $\ldots \ldots \ldots \ldots$ & -0.1604 & -0.3664 & -0.50 & +2.73 \\
\hline
\end{tabular}

\footnotetext{
${ }^{5}$ We assume a homogeneous $\Omega_{0}=1.0, \Lambda=0, H_{0}=100 h \mathrm{~km} \mathrm{~s}^{-1}$ $\mathrm{Mpc}^{-1}$ cosmology for all our calculations.
}

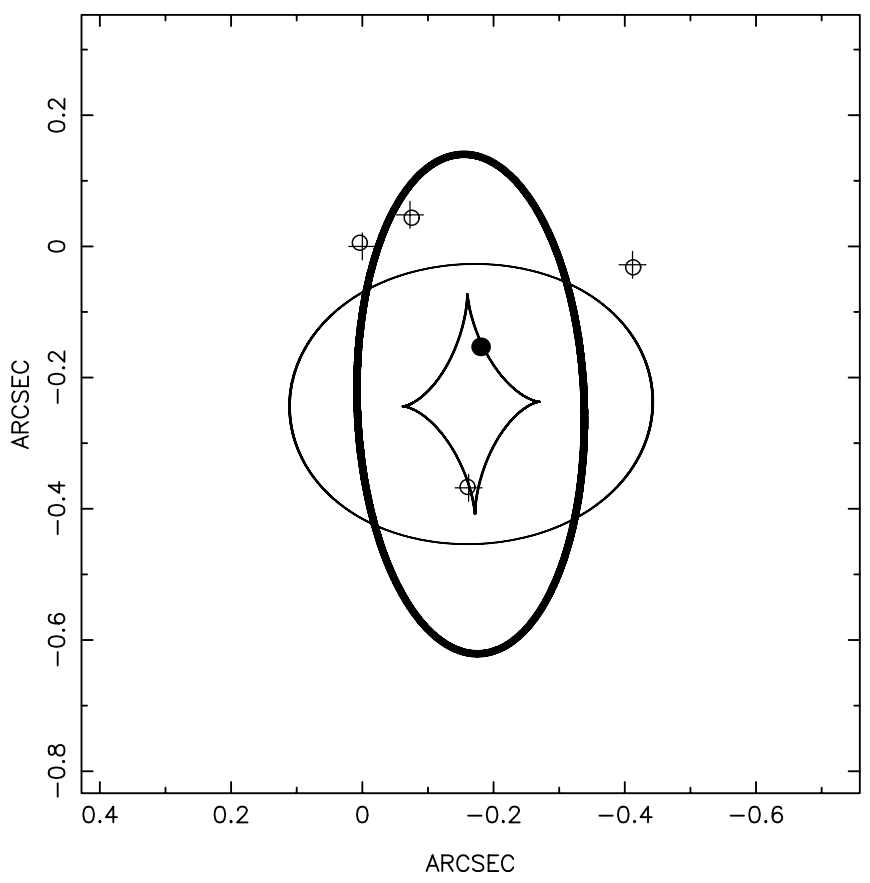

FIG. 6.- Lens model for B1555 + 375. The critical curves and caustics are shown for the SIE model. The position of the source is marked by a filled circle. The observed image positions are marked with plus signs. The best-fit model positions are marked with open circles.

more of the following: the lens galaxy is sub- $L^{*}$, it has $z>0.5$, or it is highly reddened.

\section{A LENS MODEL FOR B1555+375}

We have attempted to model the B1555+375 system using a singular isothermal ellipsoid (SIE) mass distribution (Kormann, Schneider, \& Bartelmann 1994). This model is described by seven parameters: the lens position, velocity dispersion, axial ratio, axial position angle, and the position of the source. We obtain 11 constraints (eight from the image positions and three from the flux density ratios) from the deep MERLIN $5 \mathrm{GHz}$ observation. The number of degrees of freedom (dof) is therefore four. We allowed a conservative $1 \sigma$ error of 5 mas in the relative $x$ - and $y$ positions of the image components. A conservative $1 \sigma$ error of $20 \%$ was allowed for the flux densities to account for possible variability. In the first instance, we assumed a lens redshift of $z_{l}=0.5$ and a source redshift of $z_{s}=1.5$ (consistent with values measured from other JVAS/CLASS lens systems). ${ }^{6}$

Lens modeling was performed using an image-plane minimization as described by Kochanek (1991). We optimized the goodness-of-fit parameter over all components using the positions $\left(\theta_{i}\right)$ and magnification ratios $\left[r_{i}=\right.$ $|S(i) / S(A)|]$,

$$
\chi^{2}=\sum_{i=A, B, C, D} \frac{\left(\theta_{i}^{\prime}-\theta_{i}\right)^{2}}{\Delta \theta_{i}^{2}}+\sum_{i=B, C, D} \frac{\left(r_{i}^{\prime}-r_{i}\right)^{2}}{\Delta r_{i}^{2}}
$$

where primed quantities are model-predicted and unprimed quantities are observed. A good fit $\left(\chi^{2} / \mathrm{dof}=2.6\right)$ to the

\footnotetext{
${ }^{6}$ For a summary of the JVAS/CLASS lens systems, refer to the table at http://gladia.astro.rug.nl:8000/ceres/lens_table/.
} 
MERLIN-measured positions and flux densities was obtained. The best-fit model parameters are listed in Tables 4 and 5. The critical curves, caustics, and best-fit image and source positions are displayed in Figure 6. For our assumed cosmology and lens and source redshifts, the predicted time delay between components $\mathrm{C}$ and $\mathrm{D}$ is $2.73 h^{-1}$ days. Between components $\mathrm{C}$ and $\mathrm{A}$, it is only $0.48 h^{-1}$ days.

Given the possibility of a $z_{l}>0.5$ lens galaxy, we reran the model with $z_{l}=1$ and $z_{s}=3$. With these modified redshifts, the predicted time delay between components $\mathrm{C}$ and $\mathrm{D}$ is $5.27 h^{-1}$ days. Between components $\mathrm{C}$ and $\mathrm{A}$, it is only $0.93 h^{-1}$ days. The lens velocity dispersion increases to $146.7 \mathrm{~km} \mathrm{~s}^{-1}$, and all other quantities remain unaffected.

Although this is a preliminary attempt to model B1555+375, we believe it illustrates two things: (1) the system can be modeled as a gravitational lens, and (2) the MERLIN data appear to be well described with an SIE lens without the complicating effects of a second deflector or external shear.

\section{SUMMARY AND FUTURE WORK}

We have discovered a new gravitational lens systemB1555 + 375-in the second phase of CLASS. This is clearly an atypical gravitational lens system. For the confirmed JVAS/CLASS lenses, the mean image separation is approximately 1.3 , and the distribution is sharply peaked about this value. With a maximum image separation of 0.42 , we believe B1555 + 375 to be the smallest confirmed quadruply imaged gravitational lens system.

The Keck and WHT images provide evidence for a sub- $L^{*}$ and/or high- $z$ lens galaxy. The lensed images prob- ably contribute to the total light at $H$ band; there is some sign of this in the WHT image. The red color of the system $(R-H \sim 6 \mathrm{mag})$ may also be evidence of reddening because of dust in the lensing galaxy - several confirmed lens systems show signs of dust (Jackson, Nair, \& Browne 1998a; Malhotra, Rhoads, \& Turner 1997). However, Kochanek et al. (1998) recently argued against the theory of dust in lensing galaxies and suggested that red colors are intrinsic to the lensed sources. Future Hubble Space Telescope observations of $\mathrm{B} 1555+375$ may help to resolve this conflict.

A lens mass model based on an SIE mass distribution appears to fit the MERLIN-measured image positions and flux density ratios. Our best-fit model has $\chi^{2} / \mathrm{dof}=2.6$. We predict time delays between the lensed images on the order of a few days. As a result, B1555 + 375 may only be useful in a determination of the Hubble constant if there is variability on very short timescales. Delays could be determined with a modest, densely sampled monitoring program at an angular resolution sufficient to resolve the images. Future high dynamic range VLBA observations are required to search for extended milliarcsecond-scale structures in the lensed images to further constrain the model.

We wish to thank P. J. Helbig for assistance with this work. We also thank the anonymous referee for helpful suggestions on how to improve the paper. S. T. M. is supported by an Alfred P. Sloan fellowship. This research was supported in part by the European Commission, TMR Programme, research network contract ERBFMRXCT96-0034 "CERES."
Blandford, R., \& Narayan, R. 1992, ARA\&A, 30, 311

Browne, I. W. A., Patnaik, A. R., Wilkinson, P. N., \& Wrobel, J. M. 1998, MNRAS, 293, 257

Condon, J. J., Cotton, W. D., Greisen, E. W., Yin, Q. F., Perley, R. A., Taylor, G. B., \& Broderick, J. J. 1998, AJ, 115, 1693

Douglas, J. N., Bash, F. N., Bozyan, F. A., Torrence, G. W., \& Wolfe, C. 1996, AJ, 111, 1945

Falco, E. E., Kochanek, C. S., \& Muñoz, J. A. 1998, ApJ, 494, 47

Fassnacht, C. D., et al. 1999, AJ, 117, 658

Fassnacht, C. D., Womble, D. S., Neugebauer, G., Browne, I. W. A., Readhead, A. C. S., Matthews, K., \& Pearson, T. J. 1996, ApJ, 460, L103

Gregory, P. C., \& Condon, J. J. 1991, ApJS, 75, 1011

Gregory, P. C., Scott, W. K., Douglas, K., \& Condon, J. J. 1996, ApJS, 103 , 427

Helbig, P. J., Marlow, D. R., Quast, R., Wilkinson, P. N., Browne, I. W. A., \& Koopmans, L. V. E. 1999, A\&AS, 136, 297

Jackson, N., et al. 1995, MNRAS, 274, L25

Jackson, N., Nair, S., \& Browne, I. W. A. 1998a, in Observational Cosmology with the New Radio Surveys, ed. M. N. Bremer, N. Jackson, \& I. Pérez-Fournon (Dordrecht: Kluwer), 315

Jackson, N., et al. 1998b, MNRAS, 296, 483

Kochanek, C. S. 1991, ApJ, 373, 354

Kochanek, C. S., Falco, E. E., Impey, C. D., Lehár, J., McLeod, B. A., Rix, H.-W., Keeton, C. R., \& Peng, C. Y. 1998, ApJ, submitted (astro-ph/ 9809371)
Koopmans, L. V. E., et al. 1999, MNRAS, 303, 727

Kormann, R., Schneider, P., \& Bartelmann, M. 1994, A\&A, 284, 285

Malhotra, S., Rhoads, J. E., \& Turner, E. L. 1997, MNRAS, 228, 138

Myers, S. T., et al. 1995, ApJ, 447, L5

-.1999a, AJ, 117, 2565

- $1999 \mathrm{~b}$, in preparation

Oke, J. B., et al. 1995, PASP, 107, 375

Patnaik, A. R., Browne, I. W. A., Wilkinson, P. N., \& Wrobel, J. M. 1992 MNRAS, 254, 655

Poggianti, B. M. 1997, A\&AS, 122, 399

Quast, R., \& Helbig, P. J. 1999, A\&A, 344, 721

Refsdal, S. 1964, MNRAS, 128, 307

Rengelink, R. B., Tang, Y., de Bruyn, A. G., Miley, G. K., Bremer, M. N. Röttgering, H. J. A., \& Bremer, M. A. R. 1997, A\&AS, 124, 259

Shepherd, M. C. 1997, in ASP Conf. Ser. 125, Astronomical Data Analysis Software and Systems VI, ed. G. Hunt \& H. E. Payne (San Francisco: ASP), 77

Sykes, C. M., et al. 1998, MNRAS, 301, 310

Wilkinson, P. N., Browne, I. W. A., Patnaik, A. R., Wrobel, J. M., \& Sorathia, B. 1998, MNRAS, 300, 790 